\title{
DAYTIME VEILING GLARE AND DRIVER VISUAL PERFORMANCE: INFLUENCE OF WINDSHIELD RAKE ANGLE AND DASHBOARD REFLECTANCE
}

\author{
Josef Schumann \\ Michael J. Flannagan \\ Michael Sivak \\ Eric C. Traube
}

March 1996 
DAYTIME VEILING AND DRIVER VISUAL PERFORMANCE:

INFLUENCE OF WINDSHIELD RAKE ANGLE AND DASHBOARD REFLECTANCE

\author{
Josef Schumann \\ Michael J. Flannagan \\ Michael Sivak \\ Eric C. Traube
}

The University of Michigan

Transportation Research Institute

Ann Arbor, Michigan 48109-2150

U.S.A.

Report No. UMTRI-96-13

March 1996 
Technical Report Documentation Page

\begin{tabular}{|c|c|c|c|c|}
\hline $\begin{array}{l}\text { 1. Report No. } \\
\text { UMTRI-96-13 }\end{array}$ & \multicolumn{2}{|c|}{ 2. Government Accession No. } & \multicolumn{2}{|c|}{ 3. Recipient's Catalog No. } \\
\hline \multirow{2}{*}{\multicolumn{3}{|c|}{$\begin{array}{l}\text { 4. Title and Subtitle } \\
\text { Daytime Veiling Glare and Driver Visual Performance: } \\
\text { Influence of Windshield Rake Angle and Dashboard Reflectance }\end{array}$}} & \multicolumn{2}{|c|}{$\begin{array}{l}\text { 5. Report Date } \\
\text { March } 1996\end{array}$} \\
\hline & & & \multicolumn{2}{|c|}{$\begin{array}{l}\text { 6. Performing Organization Code } \\
302753\end{array}$} \\
\hline \multicolumn{3}{|c|}{$\begin{array}{l}\text { 7. Author(s) } \\
\text { Josef Schumann, Michael J. Flannagan, Michael Sivak, and } \\
\text { Eric C. Traube }\end{array}$} & \multicolumn{2}{|c|}{$\begin{array}{l}\text { 8. Performing Organization Report No. } \\
\text { UMTRI-96-13 }\end{array}$} \\
\hline \multirow{2}{*}{\multicolumn{3}{|c|}{$\begin{array}{l}\text { 9. Performing Organization Name and Address } \\
\text { The University of Michigan } \\
\text { Transportation Research Institute } \\
2901 \text { Baxter Road } \\
\text { Ann Arbor, Michigan } 48109-2150 \text { U.S.A. }\end{array}$}} & \multicolumn{2}{|c|}{ 10. Work Unit no. (TRAIS) } \\
\hline & & & \multicolumn{2}{|c|}{ 11. Contract or Grant No. } \\
\hline \multirow{2}{*}{\multicolumn{3}{|c|}{$\begin{array}{l}\text { 12. Sponsoring Agency Name and Address } \\
\text { The University of Michigan } \\
\text { Industry Affiliation Program for } \\
\text { Human Factors in Transportation Safety }\end{array}$}} & \multicolumn{2}{|c|}{ 13. Type of Report and Period Covered } \\
\hline & & & \multicolumn{2}{|c|}{ 14. Sponsoring Agency Code } \\
\hline \multicolumn{5}{|c|}{$\begin{array}{l}\text { 15. Supplementary Notes } \\
\text { The Affiliation Program currently includes Adac Plastics, Bosch, Chrysler, Delphi Interior and } \\
\text { Lighting Systems, Ford (Automotive Components Division), GE, GM NAO Safety and Restraints } \\
\text { Center, Hella, Ichikoh Industries, Koito Manufacturing, LESCOA, Libbey-Owens-Ford, Magneti } \\
\text { Marelli, North American Lighting, Osram Sylvania, Philips Lighting, PPG Industries, Reflexite, } \\
\text { Stanley Electric, TEXTRON Automotive, United Technologies Automotive Systems, Valeo, } \\
\text { Wagner Lighting, and 3M. }\end{array}$} \\
\hline \multicolumn{5}{|c|}{ 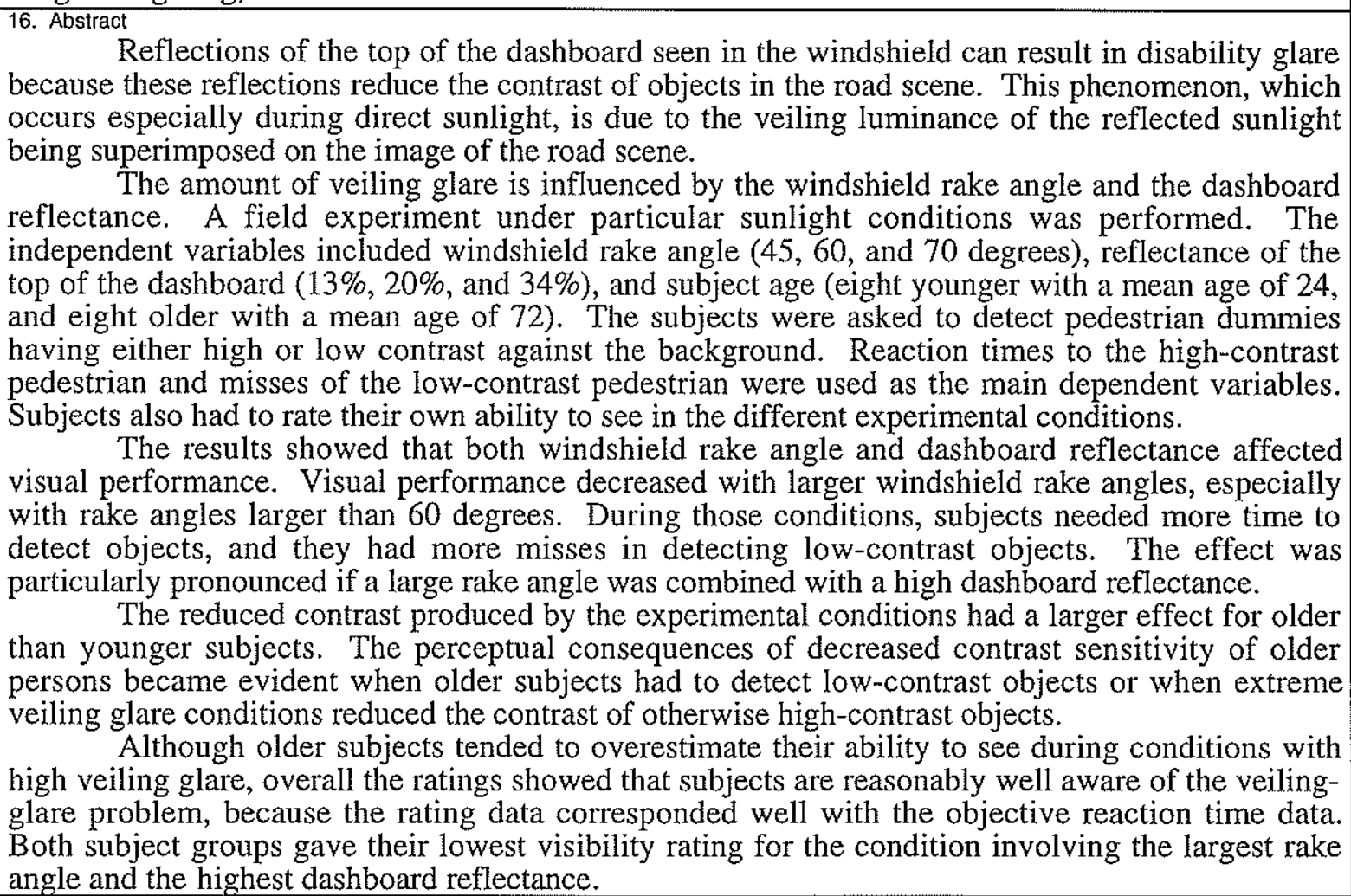 } \\
\hline & & & & \\
\hline $\begin{array}{l}\text { 19. Security Classification (of this report) } \\
\text { None }\end{array}$ & & & $\begin{array}{c}\text { 21. No. of Pages } \\
31\end{array}$ & 22. Price \\
\hline
\end{tabular}




\section{ACKNOWLEDGMENTS}

Appreciation is extended to the members of the University of Michigan Industry Affiliation Program for Human Factors in Transportation Safety for support of this research. The current members of the Program are:

Adac Plastics

Bosch

Chrysler

Delphi Interior and Lighting Systems

Ford (Automotive Components Division)

$\mathrm{GE}$

GM NAO Safety and Restraints Center

Hella

Ichikoh Industries

Koito Manufacturing

LESCOA

Libbey-Owens-Ford

Magneti Marelli

North American Lighting

Osram Sylvania

Philips Lighting

PPG Industries

Reflexite

Stanley Electric

TEXTRON Automotive

United Technologies Automotive Systems

Valeo

Wagner Lighting

$3 \mathrm{M}$ 


\section{CONTENTS}

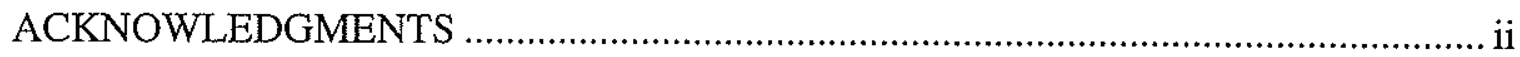

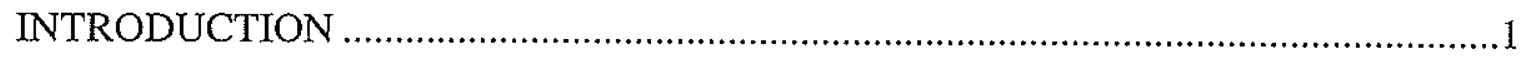

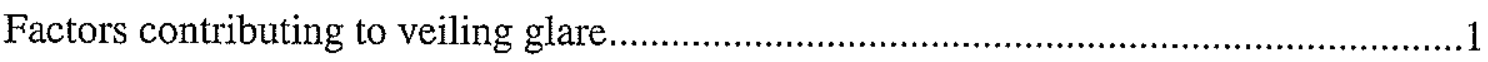

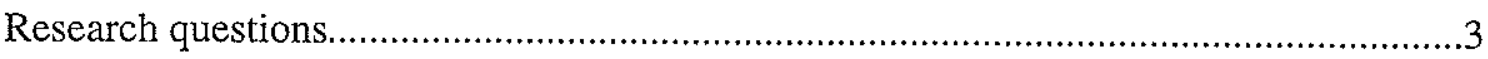

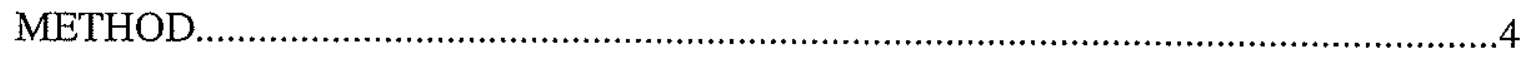

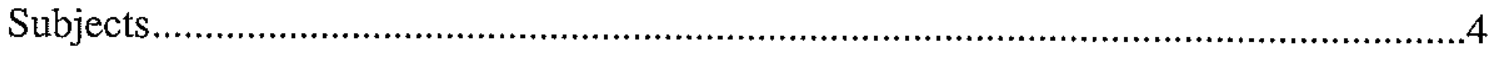

Task

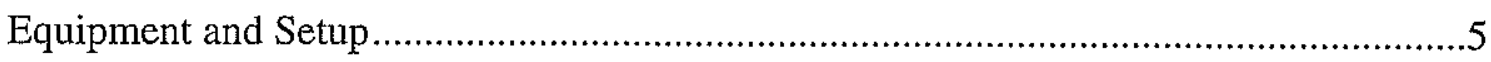

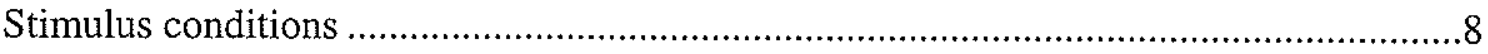

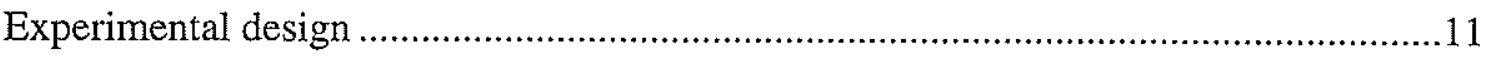

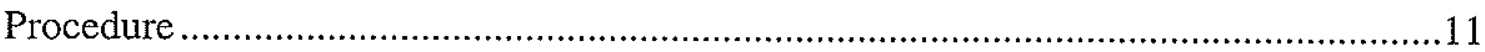

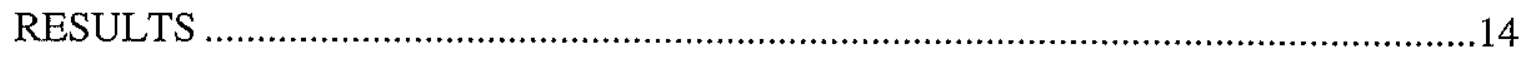

Reaction time to the high-contrast pedestrian ........................................................ 14

Misses of the low-contrast pedestrian..............................................................18

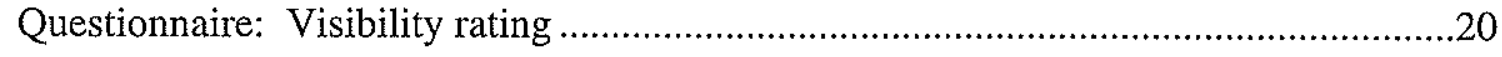

Veiling luminance as a predictor of reaction time and misses ................................23

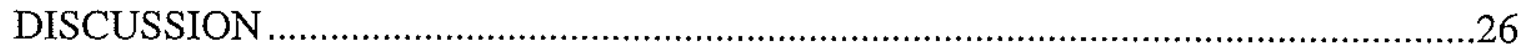

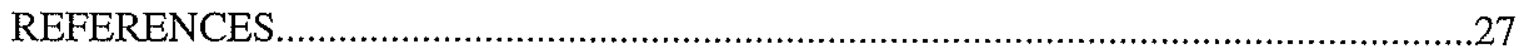




\section{INTRODUCTION}

Reflected images of the top of the dashboard seen in the windshield can impair a driver's visual performance by reducing the contrast of objects in the road scene. This phenomenon, which occurs especially during direct sunlight, is due to veiling glare from the reflected sunlight being superimposed on the image of the road scene (Kaufman \& Christenson, 1984). These reflections can produce considerable problems for drivers in certain conditions in which the reduced contrast falls below a threshold.

Although this phenomenon has not led to any legal restrictions on the use of reflecting surfaces for the top of the dashboard (49 CFR, 1994, §571.107), and is not dealt with in any SAE recommended standard practices, recently there has been growing interest in this issue from vehicle manufacturers and suppliers (Edson, 1992; Sauter, Bow, LaDriere, \& Parman, 1992; Storms, 1994).

\section{Factors contributing to veiling glare}

The amount of veiling glare is influenced by an interaction of two vehicle components, the windshield and the dashboard. The windshield mounting angle (i.e., the rake angle ${ }^{1}$ ) determines the amount of light that will be reflected by the windshield. Figure 1 shows the Fresnel reflectance (Wyszecki \& Stiles, 1982; Equation 4(1.4)), which depends on the angle of incidence (i.e., the rake angle) and the indexes of refraction of glass and air. ${ }^{2}$ Figure 1 demonstrates that the proportion of reflected light increases rapidly with windshield rake angles larger than 60 degrees. $^{3}$

\footnotetext{
The windshield rake angle is the angle between the inclined windshield and the vertical.

2 A typical index of refraction for uncoated glass is 1.5 , while for air it is 1 (Kaufman \& Christenson, 1984; p. 2-15). The refractive index ratio, representative of an air-glass boundary is thus 1.5 (Wyszecki \& Stiles, 1982; p. 53).

3 A 1990 automotive windshield light transmittance survey indicated that a substantial proportion of passenger cars and vans were equipped with windshields mounted at a rake angle of 60 degrees and more (NHTSA, 1991).
} 


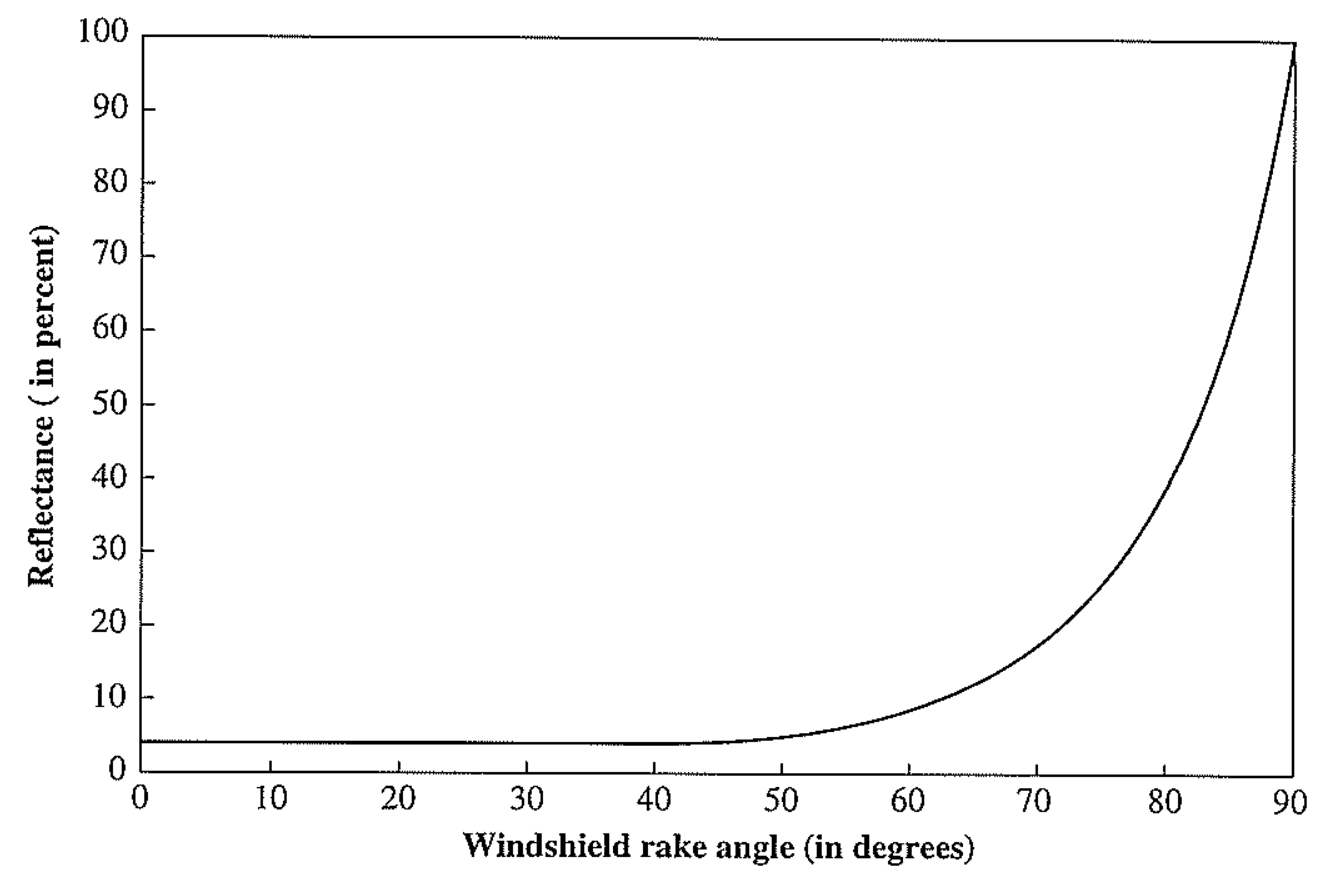

Figure 1. Fresnel reflectance as a function of angle of incidence (i.e., windshield rake angle) of a representative air-glass boundary (refractive index ratio of 1.5).

The dashboard factors influencing veiling glare are the inclination angle of the panel, lightness and gloss of the top of the panel, and high-contrast patterns (e.g., ventilator grilles) at the top of the dashboard. Gloss is defined as the property of a material surface that involves mixed reflections and is responsible for a mirrorlike appearance of the surface (Wyszecki \& Stiles, 1982). The lightness determines the percentage reflectance of the surface. The lighter the surface, the higher the reflectance. Lightness is defined on a Value scale in the Munsell classification and can be transformed to the CIE Tristimulus luminance factor Y (Wyszecki \& Stiles, 1982; Table $\Pi(6.5 .1))$. 


\section{Research questions}

Because veiling glare reduces the driver's ability to see, it has been classified as a form of disability glare. Therefore, this study was designed to investigate how different veiling glare conditions affect the driver's ability to detect objects in the road scene. Because veiling glare problems mainly occur in direct sunlight, a field experiment under particular sunlight conditions was employed, in which subjects had to detect shadowed, low-contrast objects. During the experiment, subjects had to look into a dark, tunnel-like area, surrounded by bright sunlight. This experimental scenario could be compared to real driving situations, like driving under trees and overpasses, or into tunnels and buildings.

Windshield rake angle and lightness of the top of the dashboard were chosen for experimental manipulation. Specifically, research questions concerned the following:

- the influence of windshield rake angle and dashboard top lightness on the detectability of pedestrians; and

- particular problems of older persons with veiling glare because of the known agerelated decline in contrast sensitivity (Sivak, Campbell, Schneider, Sprague, Streff, \& Waller, 1995). 


\section{METHOD}

\section{Subjects}

Sixteen paid subjects, all licensed drivers, participated in the study. There were two age groups, each consisting of eight subjects balanced by gender. Subjects were recruited from a list of people interested in participating in research studies.

The ages of subjects in the younger group ranged from 18 to 30 years (mean = 23.5 , standard deviation $=4.1)$, and in the older group from 66 to 76 years $($ mean $=72.3$, standard deviation $=5.4$ ).

All subjects wore the same eyewear, if any, that they would normally wear when driving. However, sunglasses were not permitted.

\section{Task}

Subjects were asked to perform a detection task. The task involved looking through a windshield under veiling glare conditions, and determining whether a pedestrian dummy was located on the left or right side of the interior of a semitrailer located directly in front of them. Subjects were told to respond as quickly as possible when they located the pedestrian by pressing one of two response buttons. Between trials, subjects were asked to lower their heads, without closing their eyes, and look at the top of the table in front of them.

Although a pedestrian dummy was present on every trial, this was not a forcedchoice task. Subjects were told to respond only if they saw a pedestrian. This option was introduced because pilot experiments showed that there were some veiling glare conditions during which some of the subjects (especially older subjects) repeatedly could not see the dummy pedestrian. The experimental procedure thus resembled real driving scenarios, in which drivers might not be able to see an object in the road scene because of reduced contrast. If a subject did not respond within five seconds of the trial's onset, the trial was coded as a missed response and subjects were asked to lower their heads again to prepare for the next trial. 


\section{Equipment and Setup}

Schematic diagrams of the experimental setup and the subject's view are shown in Figures 2 and 3 .

Subjects were seated outdoors, on a flatbed trailer facing east (see the upper panel of Figure 2). Subjects were seated in a height-adjustable chair while looking through an angle-adjustable windshield stand placed on top of a table in front of them (see the bottom panel of Figure 2). The simulated dashboard top was placed horizontally (a $0^{\circ}$ inclination angle). Eye position was kept approximately constant across subjects by adjusting the height of the chair so that they would always be looking through the "glare" area of the windshield, the area in which the reflected image of the sun-illuminated dashboard top appeared. After eye position was adjusted correctly, subjects were instructed always to make sure their view through the windshield would be in the same general area, and only in the glare portion. Subjects held one response button in each hand, and responded by pressing the button corresponding to the side on which they saw the pedestrian. A canopy, built to simulate the roof of a vehicle, covered the area in which the subject was seated. The canopy was attached to the windshield with black cloth, so that the windshield could be easily adjusted to different rake angles.

Two experimenters were required to run the experiment. One experimenter was in the semitrailer, normally hidden from the subject's view behind large dark panels against which the pedestrian dummies were positioned. Panels, as well as the semitrailer's walls, were covered with a dark, low-reflectance paper (dark gray, 13.5\% reflectance) so that the interior would appear relatively uniform. A small hole was drilled into one of the panels to allow the experimenter in the semitrailer to see when the subject had lowered his or her head in preparation for the next trial, so that he might change the pedestrian dummy's location and/or contrast. A second experimenter, seated behind the subject on the flatbed trailer, recorded the responses, reset the timers, and checked to make sure the subject was always looking through the glare portion of the windshield. 

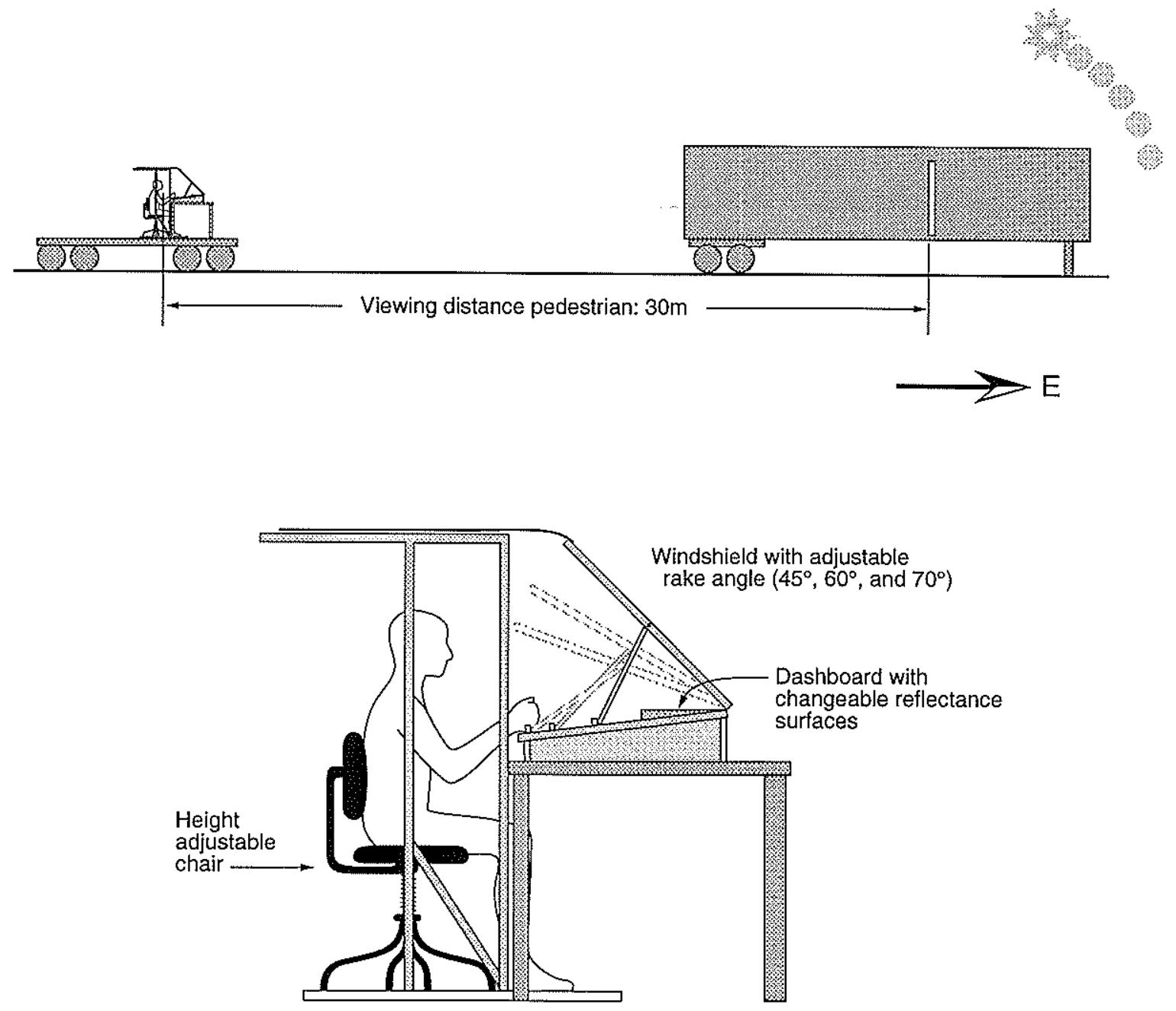

Figure 2. A schematic diagram of the experimental setup. The subject was facing east, towards the morning sun, which was usually about 30 degrees right of the subject's line of sight. 


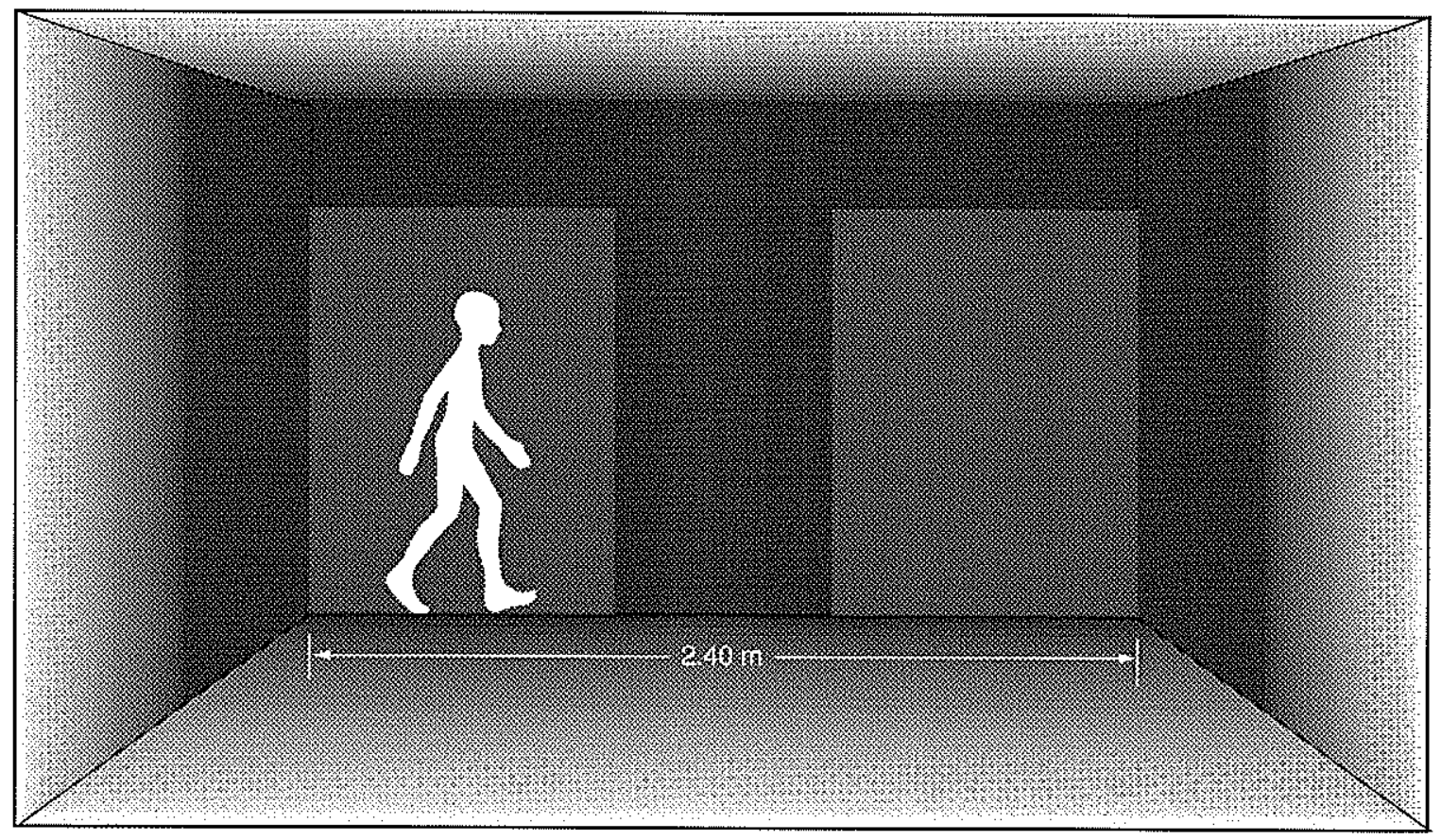

Figure 3. A schematic diagram of the subject's view, showing the high-contrast pedestrian on the left side (in one of two possible positions). Contrasts are not rendered precisely in this figure.

The location of the pedestrian dummies inside the semitrailer was $30 \mathrm{~m}$ from the subject's seated position. The width of the semitrailer's interior was $2.4 \mathrm{~m}$. Pedestrians were placed in such a way that they always appeared to be walking toward the center of the semitrailer, and were always placed in the middle of one of two large background panels (width $0.8 \mathrm{~m}$, see Figure 3 ). From the subject's position, the pedestrians were located $1.4^{\circ}$ left or right of straight ahead.

The experiment was conducted only on bright, sunny days. The equipment was positioned so that the sun was shining on the front of the windshield, from about $30^{\circ}$ to the right of the subject's line of sight. Because very few days met the study's sunshine requirements, data collection took place over six weeks. Experiment start times were adjusted to compensate for changing sunrise times throughout the time period. Four time slots were reserved for each experimental day to take into account the sun's changing position throughout morning and midday hours (before 1:00 p.m.). Figure 4 shows the varying illuminance from the sun and the sky during the testing. At the beginning of each session, measurements were taken at a horizontal surface on the table to the right of 
the windshield mockup. Subjects were approximately balanced by age and gender over the four different time slots to compensate for any sun-position effects. ${ }^{4}$

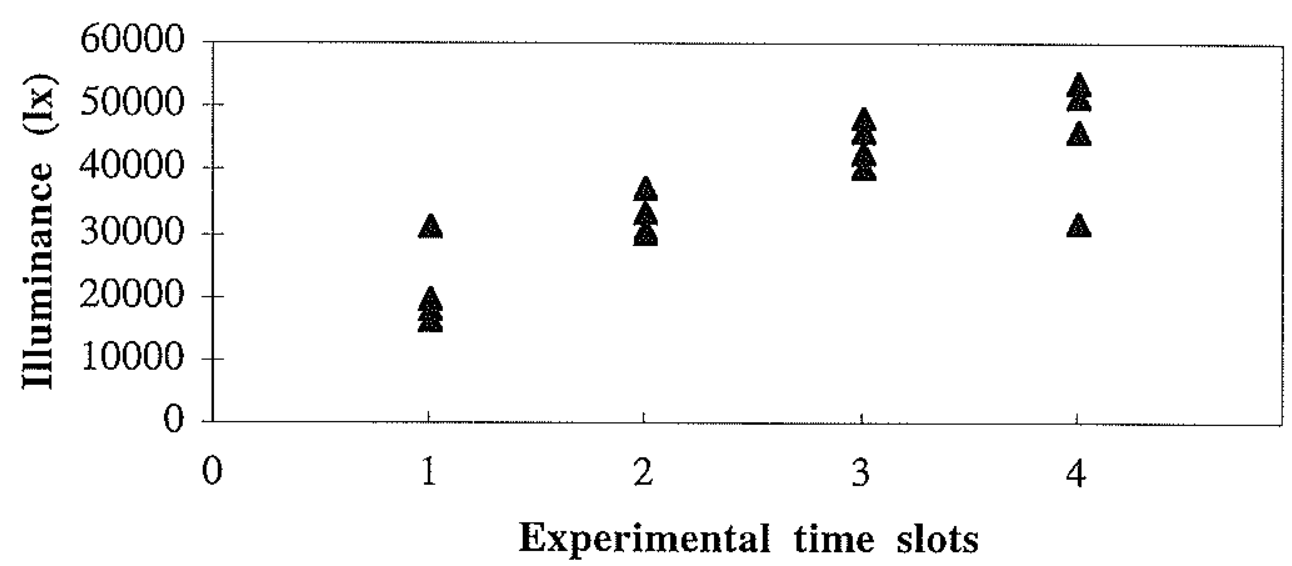

Figure 4. Illuminance from the sun and the sky at the beginning of the four possible experimental time slots (see text for details).

Two timers, connected to the subject's hand-held response buttons, were used to record response times for each trial. The experimenter positioned behind the subject would start the timers and simultaneously say "Go," which was the indication for the subject to immediately raise his or her head and begin the next trial.

\section{Stimulus conditions}

On each trial, a dummy pedestrian (height $1.25 \mathrm{~m}$ ) appeared on the left or the right side of the interior of the semitrailer. Based on a series of pilot tests, two different contrasts were chosen for the pedestrians:

- a high-contrast dummy pedestrian (average luminance contrast 6.50 ), ${ }^{5}$ which was relatively easy to detect through the veiling glare; and

- a low-contrast dummy pedestrian (average luminance contrast 1.36), which was more difficult to detect through the veiling glare.

\footnotetext{
4 Because of weather conditions, two subjects had to be tested in different time slots than planned (see the two outliers at time slots 1 and 4 in Figure 4).

5 The luminance contrast was calculated as the relationship between the luminance of the pedestrian $\left(\mathrm{L}_{\mathrm{p}}\right)$ and its background panel $\left(\mathrm{L}_{\mathrm{b}}\right)$ : $\left(\mathrm{L}_{\mathrm{p}}-\mathrm{L}_{\mathrm{b}}\right) / \mathrm{L}_{\mathrm{b}}(\mathrm{McCluney}, 1994$; Equation 2.15). The reported average was calculated from the luminance measurements taken before the start of each subject's session.
} 
Figure 5 shows the average luminance values for the background, the highcontrast pedestrian, and the low-contrast pedestrian depending on the experimental time slot during which the experiment started. The measurements were taken at the beginning of each session of the experiment, inside the semitrailer, from a distance of $3 \mathrm{~m}$.

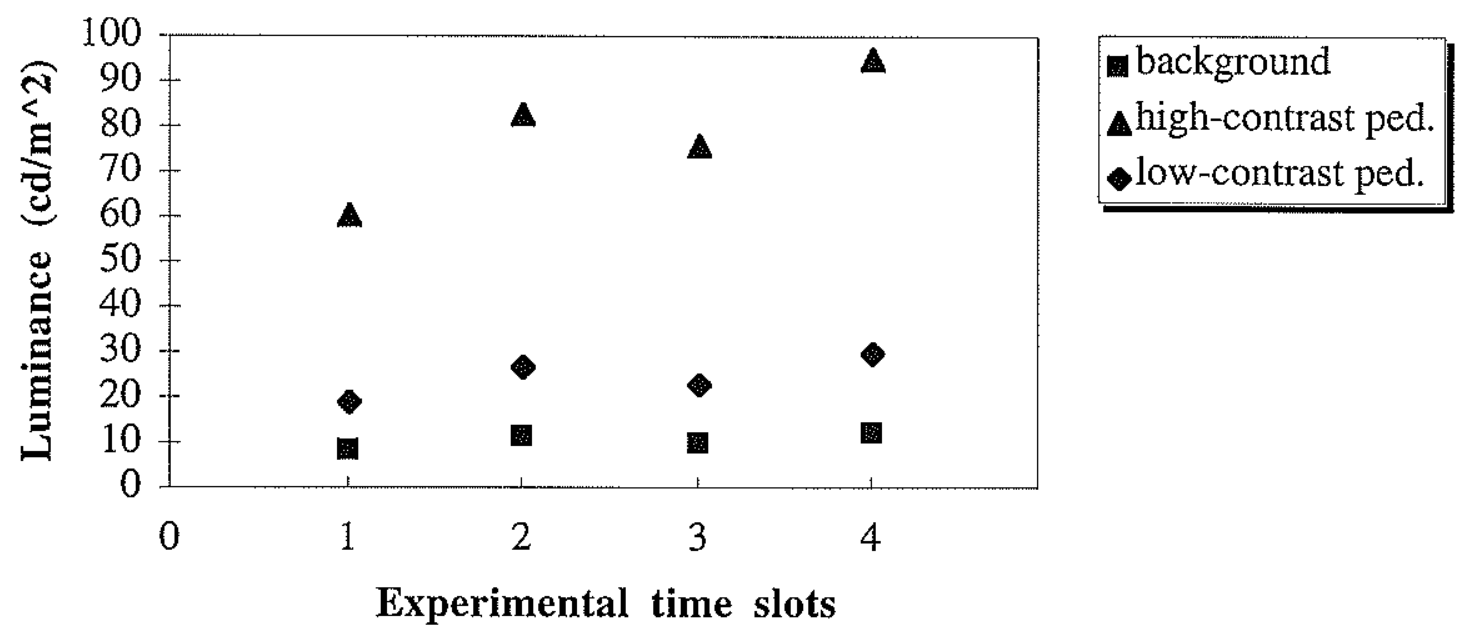

Figure 5. Average luminance measures taken inside the semitrailer at the beginning of the four possible experimental time slots.

The transmittance of the windshield for Illuminant A was approximately $77 \%$, measured normal to the glass surface.

Windshield rake angle varies considerably depending on the vehicle type. Figure 6 summarizes data from a 1990 survey of vehicle manufacturers in the United States (NHTSA, 1991). The average was calculated by weighting the windshield rake angle of each vehicle model by its sales volume. 


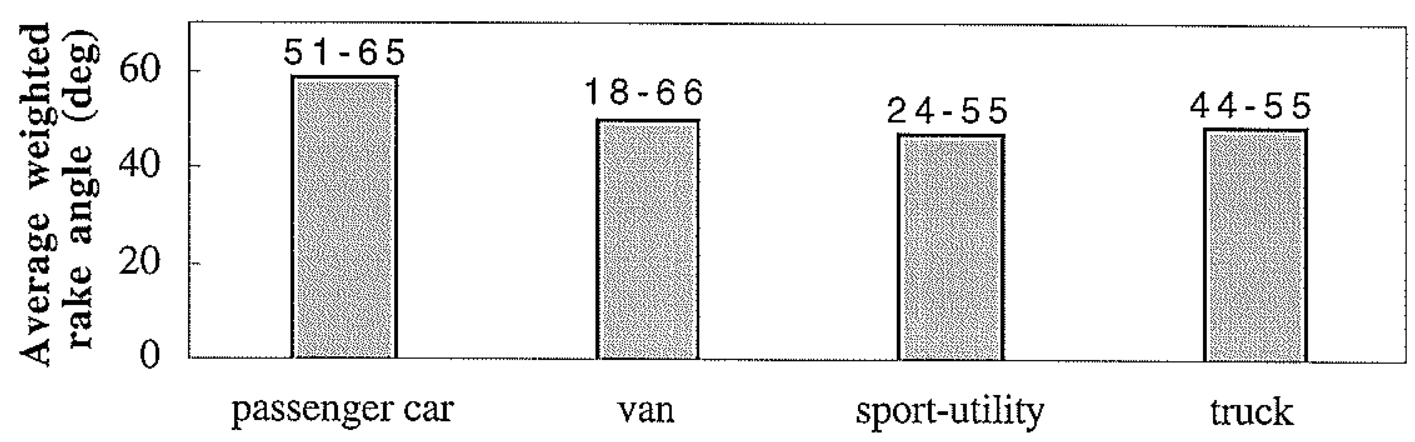

Vehicle type

Figure 6. Average windshield rake angle, weighted by sales volumes of each model, for different vehicle types in the United States (1990 data, NHTSA, 1991). Above each bar are the minimum and maximum rake angles for each vehicle type.

On the basis of the data in Figure 6, three levels of windshield rake angle were selected for this experiment:

- $45^{\circ}$, near the average for sport-utility vehicles,

- $60^{\circ}$, near the average for passenger cars, and

- $70^{\circ}$, a realistic upper limit for windshield rake angles.

For each subject, three new dashboard tops for each of the tested reflectances were used to prevent possible bleaching by sunlight. Measurements of the used material did not show any changes in reflectance over a time period of one hour, the total experimental time for each subject. Each dashboard top was used for only about 10 minutes.

Three levels of dashboard reflectance were used. Reflectance was measured at three different locations on each dashboard top and found to be uniform. Reflectances for the three levels of material were:

- low (reflectance.13; Munsell Value 4.2),

- medium (reflectance .20; Munsell Value 5.1), and

- high $^{6}$ (reflectance .34; Munsell Value 6.3).

Table 1 summarizes the experimental conditions used to manipulate veiling glare.

- The high reflectance level lies slightly above an outdated Federal purchasing standard (Fed. Std. No. 515/3a, 1967), which restricted the luminous directional reflectance of instrument panel top surfaces to a maximum of $30 \%$ (Munsell Value 6). 
Table 1. Levels of independent variables used to manipulate veiling glare. The nine experimental conditions were produced by factorially combining these levels.

\begin{tabular}{|l|l|}
\hline \multicolumn{1}{|c|}{ Variable } & \multicolumn{1}{c|}{ Levels } \\
\hline Dashboard reflectance & low $(13.5 \%)$, medium $(20.2 \%)$, and high $(34.3 \%)$ \\
\hline Windshield rake angle & $45^{\circ}, 60^{\circ}$, and $70^{\circ}$ \\
\hline
\end{tabular}

\section{Experimental design}

The following independent variables were factorially combined:

(1) age, a between-subjects variable with two levels (younger, older),

(2) windshield rake angle, a within-subjects variable with three levels $\left(45^{\circ}\right.$, $60^{\circ}$, and $70^{\circ}$ ), and

(3) dashboard reflectance, a within-subjects variable with three levels (low, medium, high).

There were nine combinations of rake angle and dashboard reflectance. They were presented in blocks of trials, with the order of these blocks randomized for each subject. Within each block, the two levels of pedestrian contrast (low and high) were presented six times each, positioned equally often against the left or the right background panel. The order of the 12 trials within each block was randomized.

\section{Procedure}

Subjects were instructed to lower their heads between trials without closing their eyes, and look at the tabletop in front of them until the experimenter behind them said "Go" to begin the next trial. When the subjects heard that signal, they were to raise their heads quickly, look through the glare portion of the windshield, and determine whether there was a pedestrian dummy on the left or right side of the interior of the semitrailer in front of them. They were told to press the left button if the dummy was on the left, and to press the right button if the dummy was on the right. They were further instructed that if they did not see a pedestrian, they were not to respond at all. If the subjects did not respond within five seconds, the experimenter would then tell them to lower their heads and prepare for the next trial.

The subjects were asked to raise their heads in a quick, consistent manner for each trial, making sure that they only looked through the glare portion of the windshield. Some practice in raising their heads on the experimenter's signal was given before the 
experiment began, so that subjects knew what would be expected of them. They were asked not to move their heads or eyes outside of the glare area for any reason. The experimenter on the flatbed trailer, as well as the experimenter in the semitrailer (looking through the small hole in the panel) watched subjects to make sure they did not deviate from the instructions given.

Two dependent variables were recorded:

(1) reaction time, measured from when the experimenter said the word "Go" until the subject pressed one of the buttons, and

(2) missed and incorrect responses.

Subjects were not told whether their responses were correct or incorrect. Any response longer than five seconds was recorded as a "missed response." Short breaks were given between blocks, during which the experimenter would change the windshield angle and/or dashboard top.

A short questionnaire (see Figure 7) was given to the subjects after each block of trials, asking them to indicate whether it was difficult, medium, or easy to discern the pedestrians, and then to quantify their answer on a numerical scale. The questionnaire is a modified version of a two-level sequential judgment scale (Pitrella \& Käppler, 1988; Käppler, Godthelp, v.Randwijk, \& Ruijs, 1992), designed to produce reliable and equalinterval data for the use of parametric statistics. 


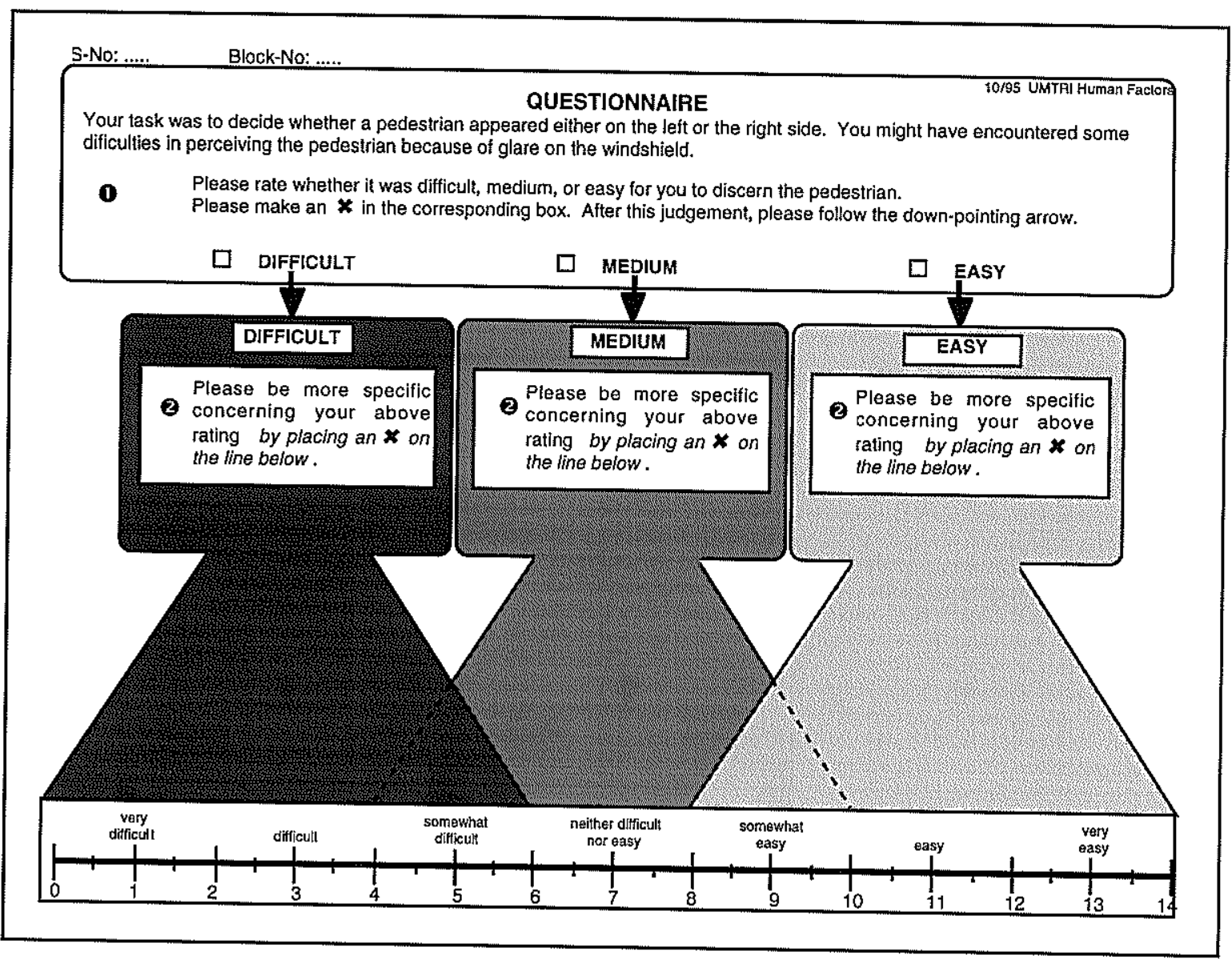

Figure 7. Two-level sequential rating scale. 


\section{RESULTS}

Two analyses were performed:

(1) an analysis of the subjects' reaction time to the high-contrast pedestrian,

(2) an analysis of the subjects' misses or incorrect responses to the lowcontrast pedestrian.

Both measures can be taken as indices of how veiling glare influences drivers' ability to see, and possibly their decisions during driving. Reaction time to the highcontrast pedestrian is a sensitive index of how veiling glare can degrade visibility of detectable objects, and misses of the low-contrast pedestrian provide a more appropriate measure for the effects of veiling glare in a more difficult situation.

Finally, the results of the subjective visibility ratings were analyzed to get information about the relation between subjective impressions of glare and objective visual decrements.

\section{Reaction time to the high-contrast pedestrian}

There were six high-contrast trials (three repetitions for each of the two positions of the pedestrian) in each of the nine experimental conditions. Correct responses were combined to an average reaction time for each subject. There were only 13 misses or incorrect responses (1.5\%) to the high-contrast pedestrian for all 16 subjects. As expected, most of misses and incorrect responses (12) were made by older subjects $(2.8 \%)$, and only one by a younger subject $(0.2 \%)$. If a subject had a miss or an incorrect response to the high-contrast pedestrian, the remaining correct responses were used to derive the average reaction time.

To take into account possible outliers for the reaction-time responses, both means and medians were calculated for the reaction-time measure. Separate analyses of variance for those measures led to the same results. Only the analyses based on mean reaction time are presented here. The analysis of variance incorporated one betweensubjects variable (age group) and two within-subjects variables (windshield rake angle and dashboard reflectance).

Two main effects were significant, with high windshield rake angle and high dashboard reflectance both resulting in longer reaction times. Figure 8 shows the significant effect of windshield rake angle, $F(2,28)=27.77, p<.0001$. A post-hoc 
analysis revealed that the only significant differences were those between the $45^{\circ}$ and $70^{\circ}$ rake angles, and between the $60^{\circ}$ and $70^{\circ}$ rake angles, $\operatorname{HSD}^{7}(3,28)=0.23 \mathrm{~s}, \alpha=.05$.

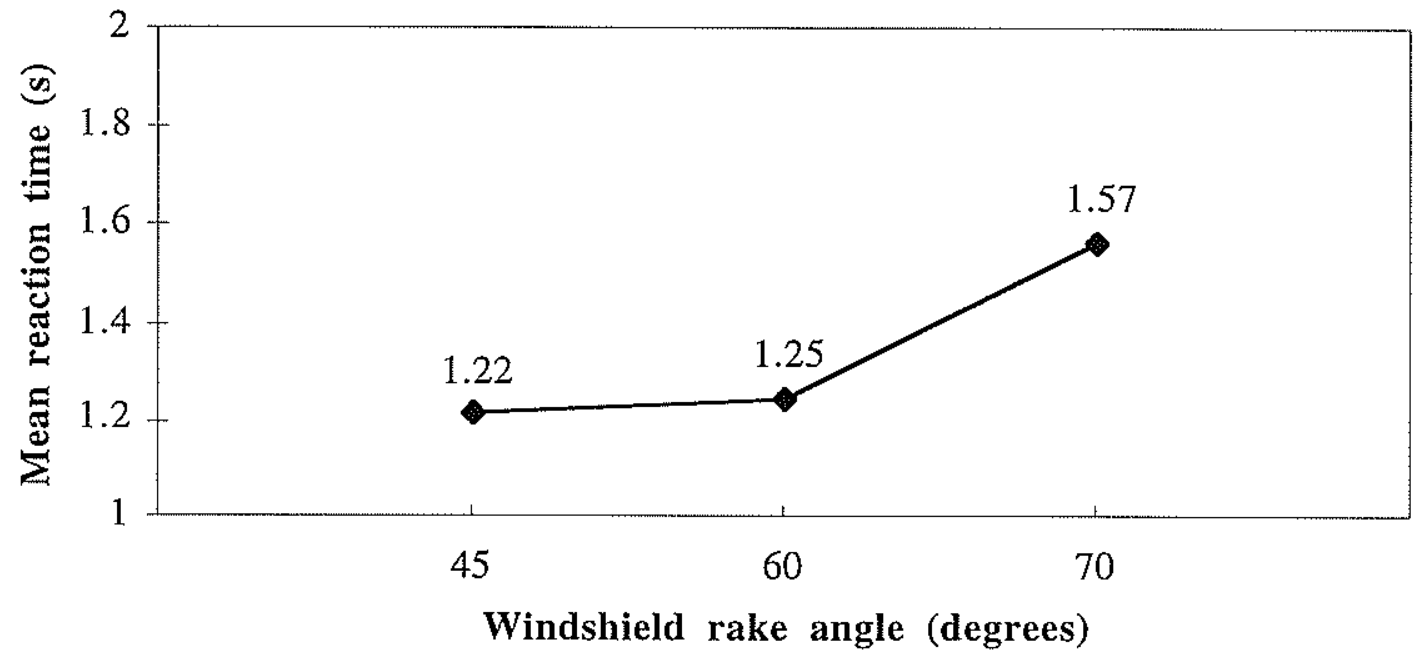

Figure 8. Mean reaction time by windshield rake angle.

Figure 9 shows the significant influence of dashboard reflectance on the subjects' reaction times, $F(2,28)=17.99, p<.0001$. The only significant differences were those between the low and high reflectances, and between the medium and high reflectances, $\operatorname{HSD}(3,28)=0.16 \mathrm{~s}, \alpha=.05$.

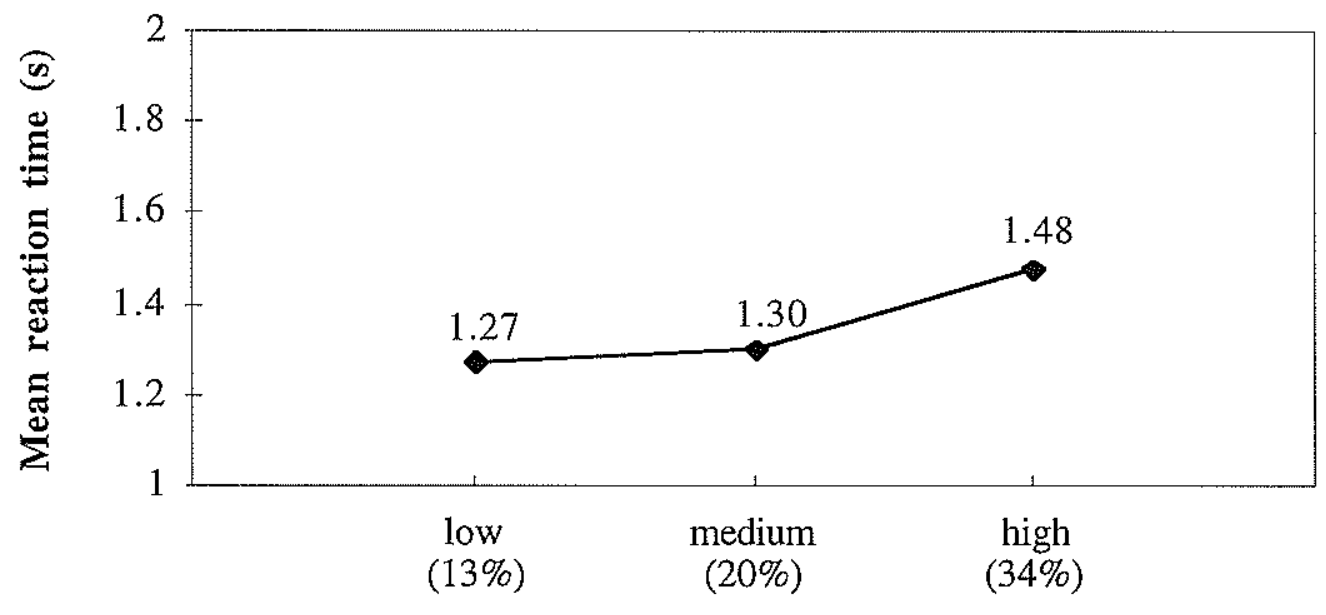

Dashboard reflectance

Figure 9. Mean reaction time by dashboard reflectance.

7 HSD: Tukey's Honestly Significant Difference test (Kirk, 1982). 
The mean reaction time of older subjects was $430 \mathrm{~ms}$ longer than the mean reaction time of younger subjects, but this difference was not statistically significant, $F(1,14)=4.12, p=.06$. However, second-order interactions involving age showed that, compared with younger subjects, the older subjects' responses tended to be affected by the $70^{\circ}$ rake angle (rake angle $\mathrm{x}$ age interaction, $F(2,28)=3.29, p=.052$ ), but not by the high-reflectance dashboard (dashboard reflectance $\mathrm{x}$ age interaction, $F(2,28)=0.91, p=$ $.42)$.

Figure 10 depicts the significant rake angle $\mathrm{x}$ dashboard reflectance interaction, $F(4,56)=12.75, \mathrm{p}<.0001$. It demonstrates that a large rake angle combined with a high dashboard reflectance produced veiling glare that resulted in especially long reaction times, $\operatorname{HSD}(9,56)=0.29 \mathrm{~s}, \alpha=.05$. On the other hand, the data showed that a low dashboard reflectance can compensate for the disadvantages of a large windshield rake angle (see Figure 10). The reaction times for the $70^{\circ}$ rake angle, low or medium dashboard-reflectance conditions were statistically not different from those for the $45^{\circ}$ and $60^{\circ}$ rake angles coupled with any tested dashboard-reflectance condition.

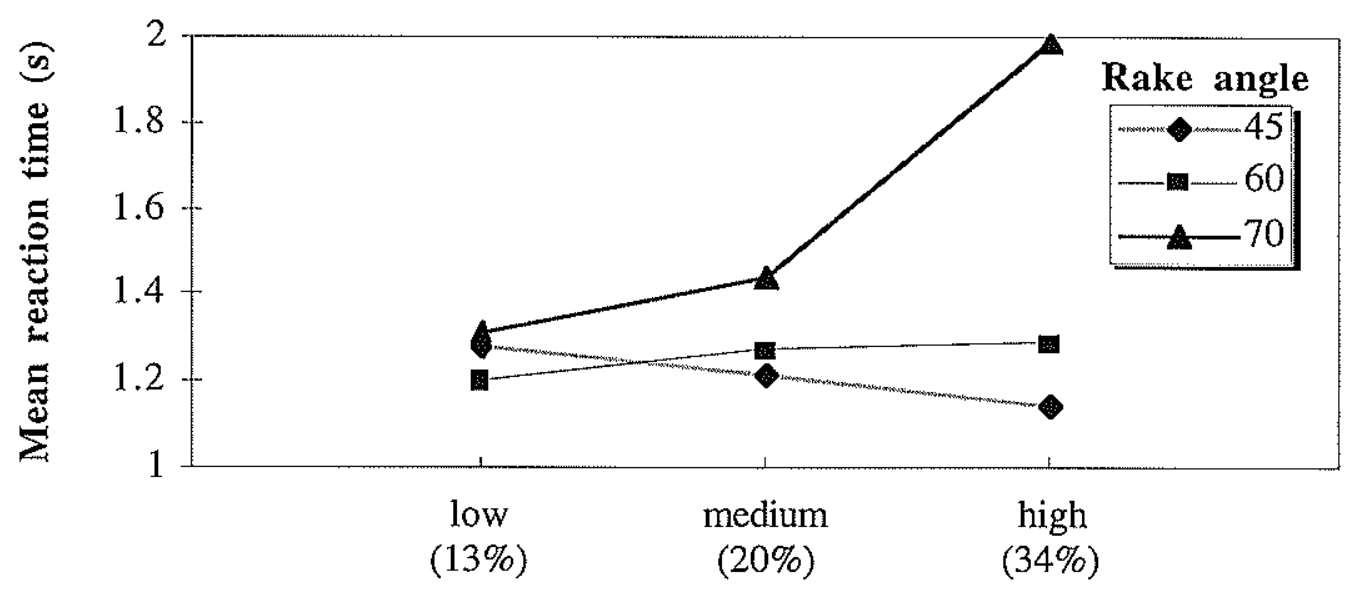

Dashboard reflectance

Figure 10. Mean reaction time by windshield rake angle and dashboard reflectance. 
The combination of the largest rake angle and highest dashboard reflectance was especially difficult for older drivers (see Figure 11). This is evident in a significant threeway interaction between age, rake angle, and dashboard reflectance, $F(4,56)=3.62, p=$ .01 .

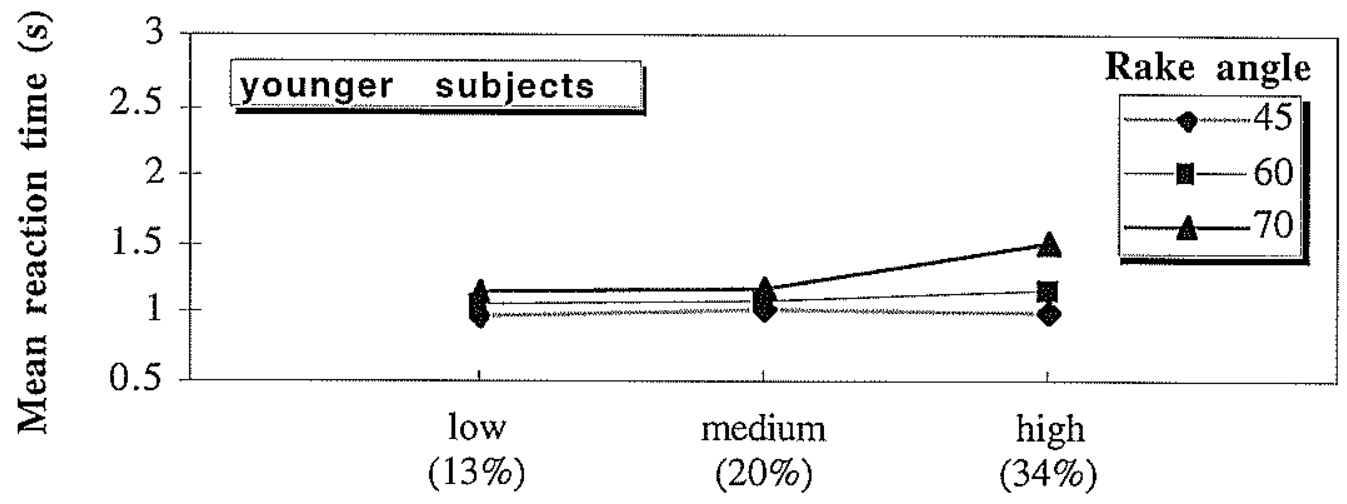

Dashboard reflectance

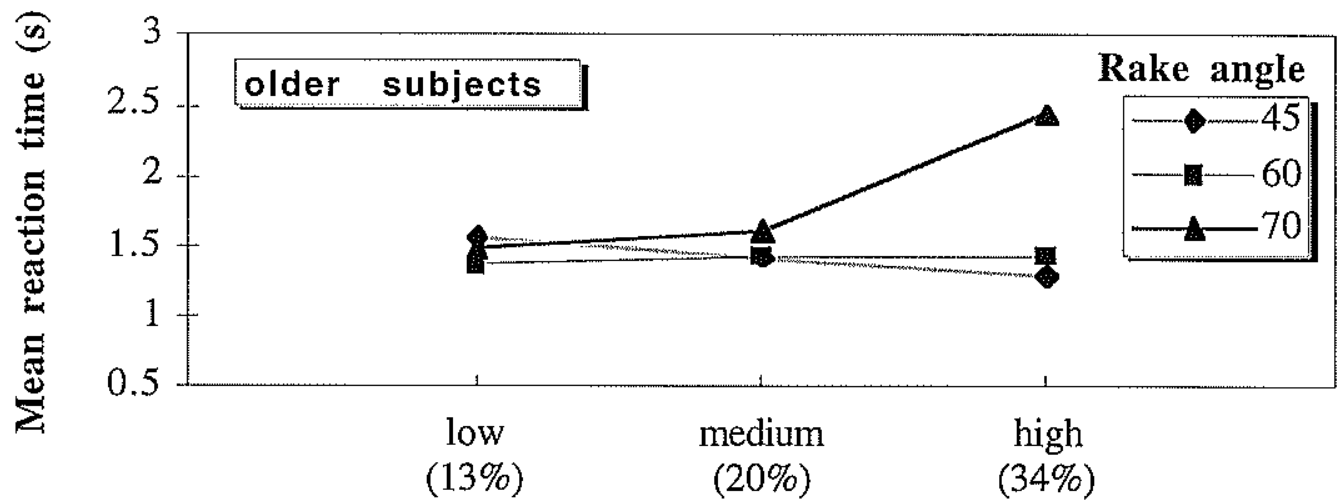

Dashboard reflectance

Figure 11. Mean reaction time by rake angle and dashboard reflectance (top panel: younger subjects; bottom panel: older subjects). 


\section{Misses of the low-contrast pedestrian}

Misses and incorrect responses with the low-contrast pedestrian were combined into a category of "misses," because there were only three incorrect responses $(0.1 \%)$. The number of all misses out of six repeated trials for each tested condition was taken as an index of how difficult it was to detect the low-contrast pedestrian. A mixed-factor analysis of variance was performed on those misses. The ANOVA incorporated one between-subjects variable (age group) and two within-subjects variables (windshield rake angle and dashboard reflectance).

All three main effects, but no interactions, were statistically significant. It was much more difficult for older subjects to detect the low-contrast pedestrian, and misses increased for both age groups with larger windshield rake angles and higher dashboard reflectance.

Figure 12 indicates the significant influence of age on the misses, $F(1,14)=$ $11.60, p=.004$. Older subjects had particular difficulties for the $70^{\circ}$ rake angle and high dashboard-reflectance combination ( $94 \%$ misses).

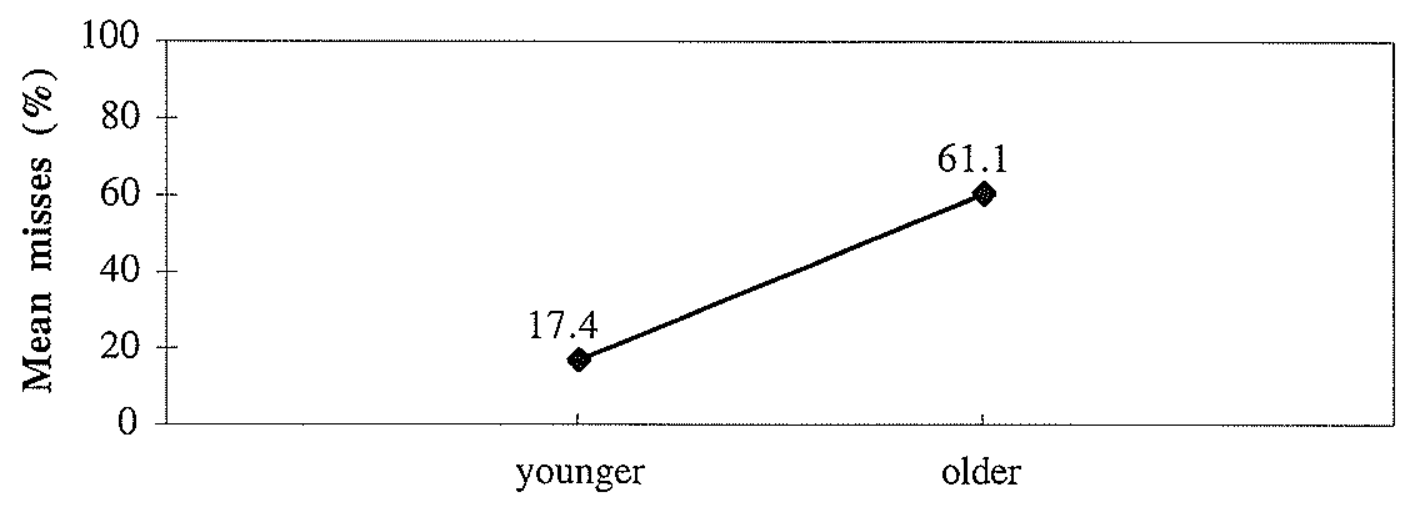

Age group

Figure 12. Percentage of misses by age group. 
Figure 13 and 14 show the significant influence on misses of windshield rake angle, $F(2,28)=19.17, p<.0001$, and dashboard reflectance $F(2,28)=6.08, \mathrm{p}=.013$, respectively. A post-hoc analysis revealed that the only significant differences were those between the $45^{\circ}$ and $70^{\circ}$ rake angles, and between the $60^{\circ}$ and $70^{\circ}$ rake angles, $\operatorname{HSD}(3,28)=30.2 \% ; \alpha=.05$.

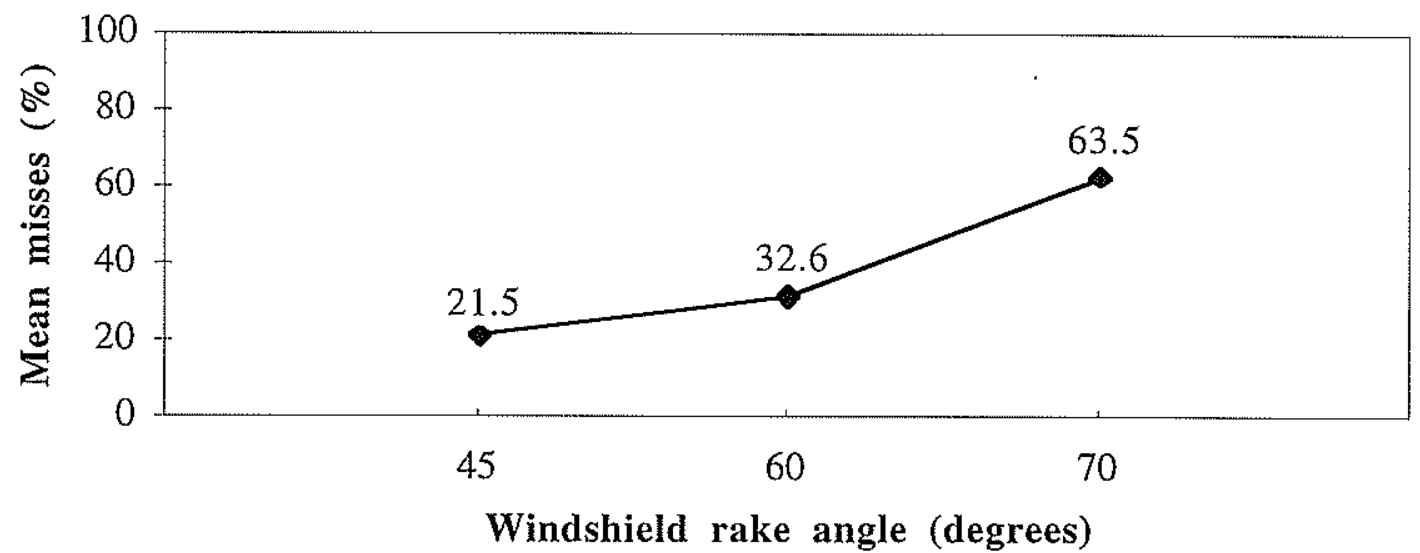

Figure 13. Percentage of misses by windshield rake angle.

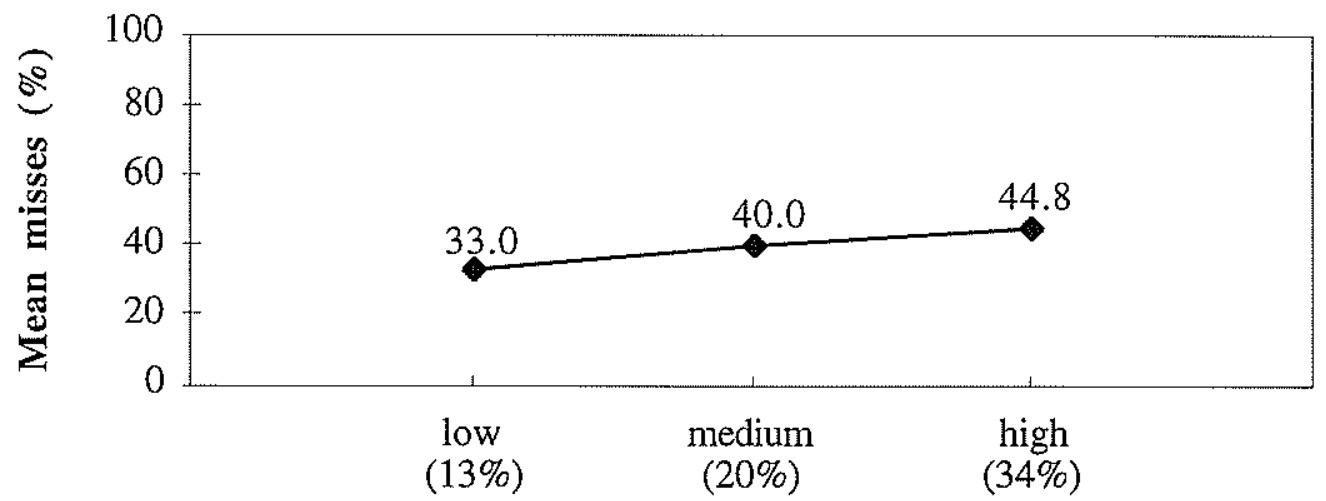

Dashboard reflectance

Figure 14. Percentage of misses by dashboard reflectance. 


\section{Questionnaire: Visibility rating}

After subjects had finished a block of 12 trials with a particular combination of rake angle and dashboard reflectance, they rated how the veiling glare affected their ability to detect pedestrians (see Figure 7). Data were coded into integers from 0 to 14 using the following rule:

- $0 \leq$ rating $<1 \Leftrightarrow$ rating code 0

- $1 \leq$ rating $<2 \Leftrightarrow$ rating code 1

- $2 \leq$ rating $<3 \Leftrightarrow$ rating code 2 ;

- .

- .

- $13 \leq$ rating $<14 \Leftrightarrow$ rating code 13 ;

- 14 = rating $\Leftrightarrow$ rating code 14

Because subjects were not told that pedestrians were present on each trial, their rating was based only on their perceived ability to see the pedestrians. Therefore, the ratings might have been biased to the extent that for the difficult experimental conditions (which resulted in poor visibility of the low-contrast pedestrian), subjects might have rated only their ability to see the high-contrast pedestrian. The data indicate that older subjects frequently could not see the low-contrast pedestrian (see Figure 12). Therefore, they could have overestimated their ability to see in the experimental conditions involving higher levels of veiling glare. Such a trend can be seen in Figure 15, in which the older subjects (compared with the younger subjects) rate their visibility as being less impaired when the windshield rake angle is increased.

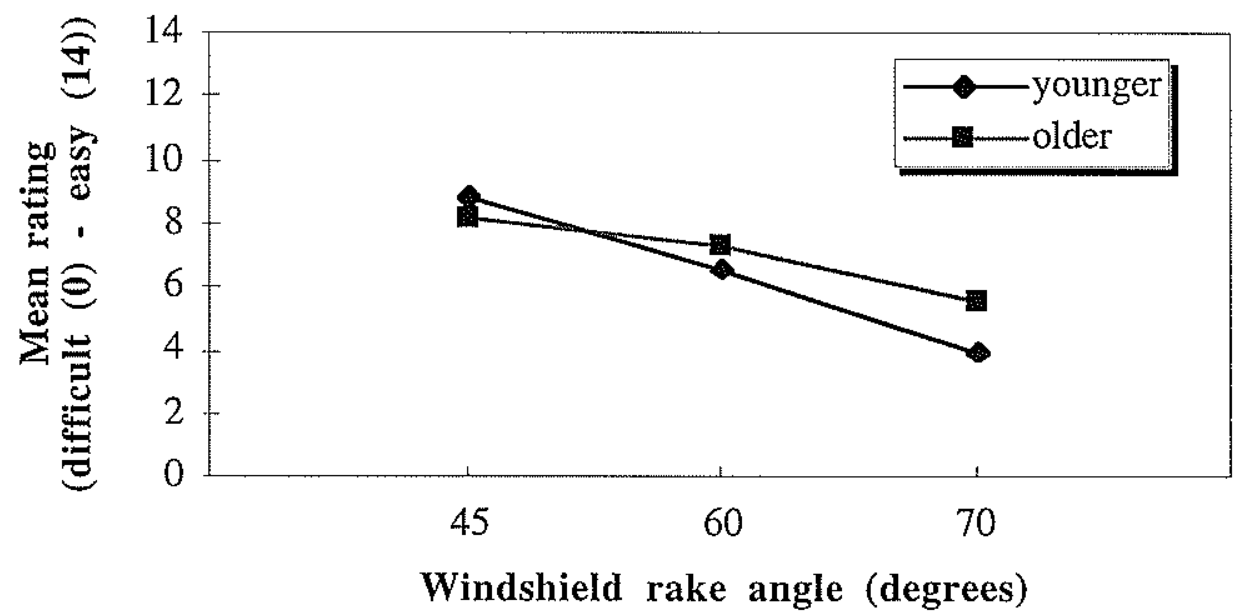

Figure 15. Mean visibility rating by age group. 
Because older subjects may have used a different decision criterion for their visibility ratings, separate repeated-measures analyses of variance were performed for each age group, with windshield rake angle and dashboard reflectance as independent variables. ${ }^{8}$

For the younger subjects, the only significant effect was windshield rake angle, $F(2,14)=21.94, p<.0001$. As expected based on the objective visibility data, younger subjects rated their ability to see to be worse with larger windshield rake angles. For the older subjects, both main effects were significant, windshield rake angle, $F(2,14)=4.2$, $p=.04$, and dashboard reflectance, $F(2,14)=4.1, p=.04$. The mean ratings by rake angle and dashboard reflectance are shown in Figure 16 for the younger subjects and in Figure 17 for the older subjects.

8 Analyses of variance were performed with the rating data, because the employed scale allows the use of parametric statistics (see Käppler et al., 1992). 


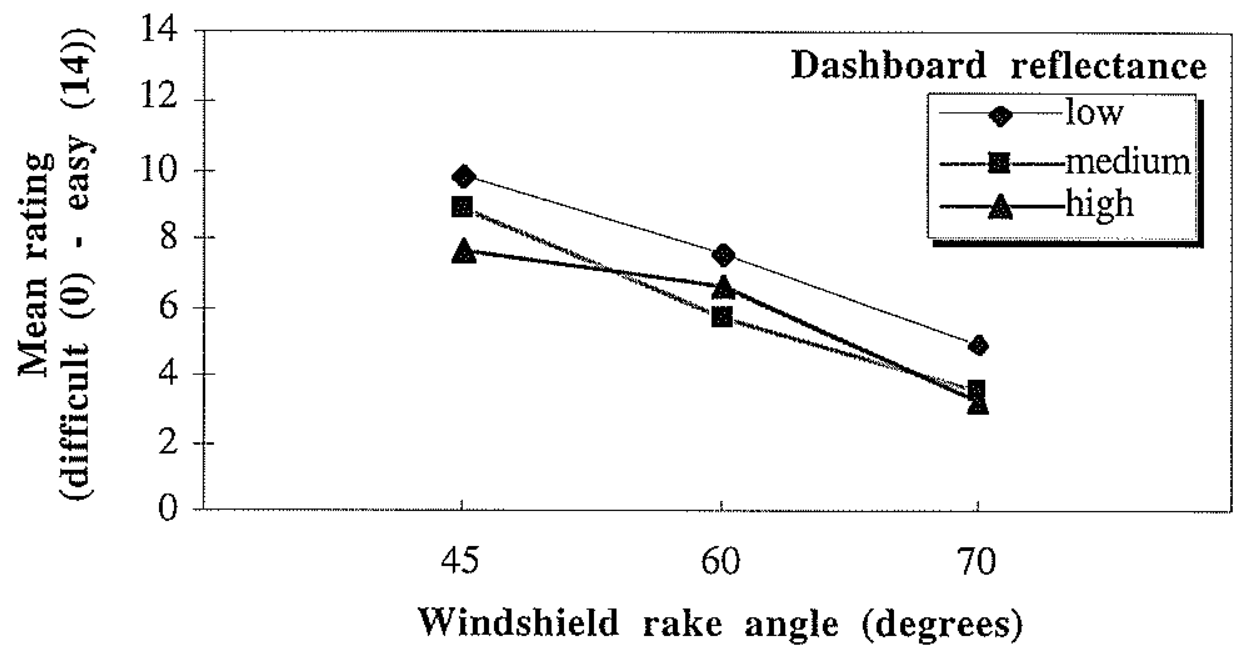

Figure 16. Mean visibility rating for younger subjects by windshield rake angle and dashboard reflectance.

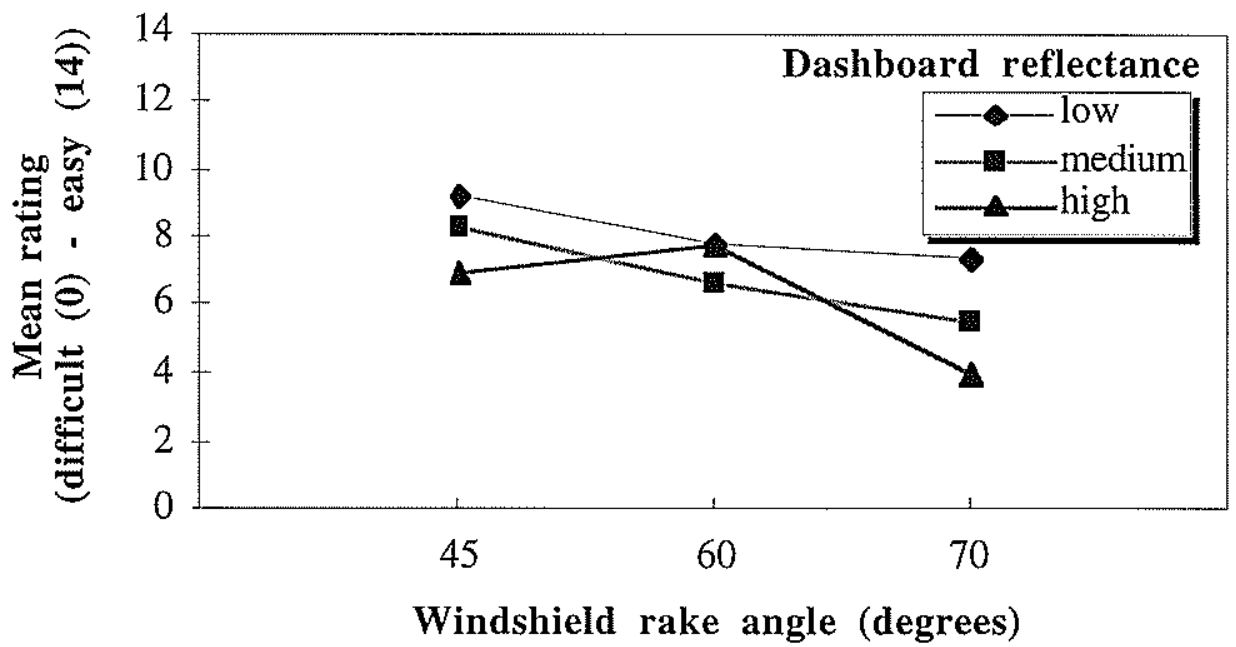

Figure 17. Mean visibility rating for older subjects by windshield rake angle and dashboard reflectance. 


\section{Veiling luminance as a predictor of reaction time and misses}

We have discussed the effects of windshield rake angle and dashboard reflectance, and their interaction, on reaction time and error rates in empirical terms. However, it is reasonable to suppose that rake angle and dashboard reflectance have their effects on the performance measures through their joint effect on veiling luminance, as depicted in Figure 18. In this section we derive a measure of veiling luminance, and demonstrate that this intervening variable accounts well for the joint effects of rake angle and dashboard reflectance, including the interaction seen in Figure 10.

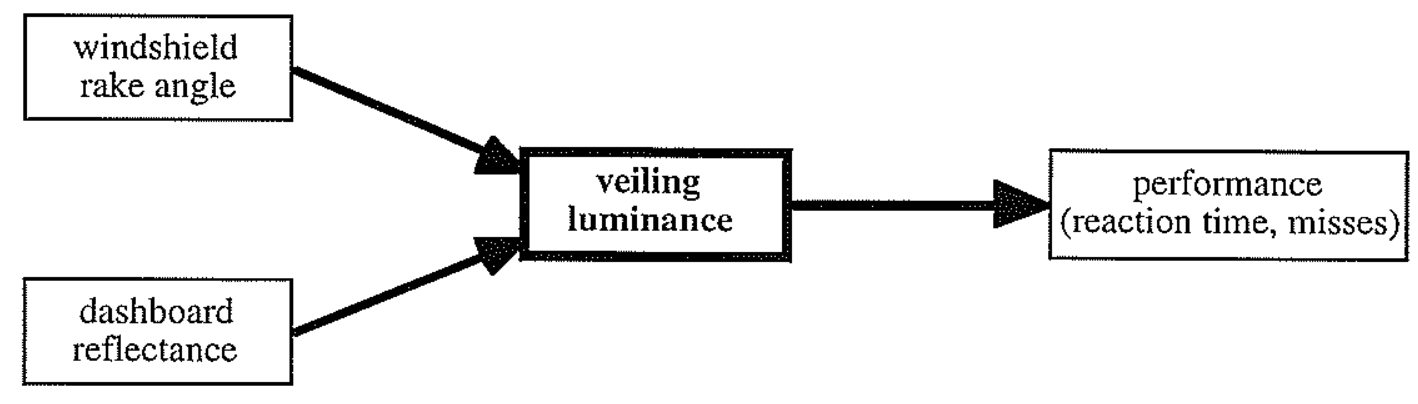

Figure 18. A simple model of the relationship between windshield rake angle, dashboard reflectance, the intervening variable (veiling luminance), and performance.

The reflected image of the dashboard appears to the subject to be just beyond the windshield, in the subject's line of sight to the pedestrians. The luminance of this image can be predicted approximately from the Fresnel reflectance at the windshield rake angle (see Figure 1) and the luminance of the dashboard (see Equations 1 and 2).

$$
\begin{aligned}
& L_{\mathrm{V}}=R(\theta) \times L_{\mathrm{D}}\left(E, \rho_{\mathrm{s}}\right) \\
& L_{\mathrm{D}}\left(E, \rho_{\mathrm{s}}\right)=\left(E \times \rho_{\mathrm{s}}\right) / \pi^{9}
\end{aligned}
$$

where $L_{v}$ is veiling luminance, $\theta$ is windshield rake angle, $R(\theta)$ is Fresnel reflectance (see Wyszecki \& Stiles, 1982; Equation $4(1.4)$ ), $L_{\mathrm{D}}$ is the luminance of the dashboard, $E$ is illuminance from the sun and the sky, and $\rho_{s}$ is dashboard reflectance.

$9 \quad$ See Wyszecki and Stiles (1982; Equation 8(4.3.3)). 
A constant illuminance of $36,000 \mathrm{~lx}$ was chosen, representing the average illuminance value measured over the four experimental time slots (see Figure 4). The average transmittance of the windshield for direct sunlight reaching the dashboard was $75 \%$ (averaged over the three experimental rake angles). This is as expected because direct sunlight was usually nearly normal to the surface of the windshield. The illuminance through the windshield was therefore set at 27,000 1x.

Figure 19 shows the relationship between the calculated veiling luminance and reaction time to the high-contrast pedestrian (top panel), and misses of the low-contrast pedestrian (bottom panel).

Figures 19 indicates strong linear relationships between veiling luminance, calculated according to Equation 1, and both reaction time and misses. Linear regression analyses show reasonably good fits, especially for younger subjects (see the $\mathrm{R}^{2}$ values for both age groups in Figure 19).

When the combined effects of rake angle and dashboard reflectance on veiling luminance are considered, it is obvious why the combination of the largest rake angle and the highest dashboard reflectance might lead to particularly long reaction times, as shown in Figure 10. In terms of veiling luminance, this combination is much higher than the other eight combinations of rake angle and dashboard reflectance. Rather than being an anomaly, as it might appear in Figure 10, the long reaction times for the combination of largest rake angle and highest reflectance are, as shown in Figure 19, part of a simple relationship between reaction time and veiling luminance. 

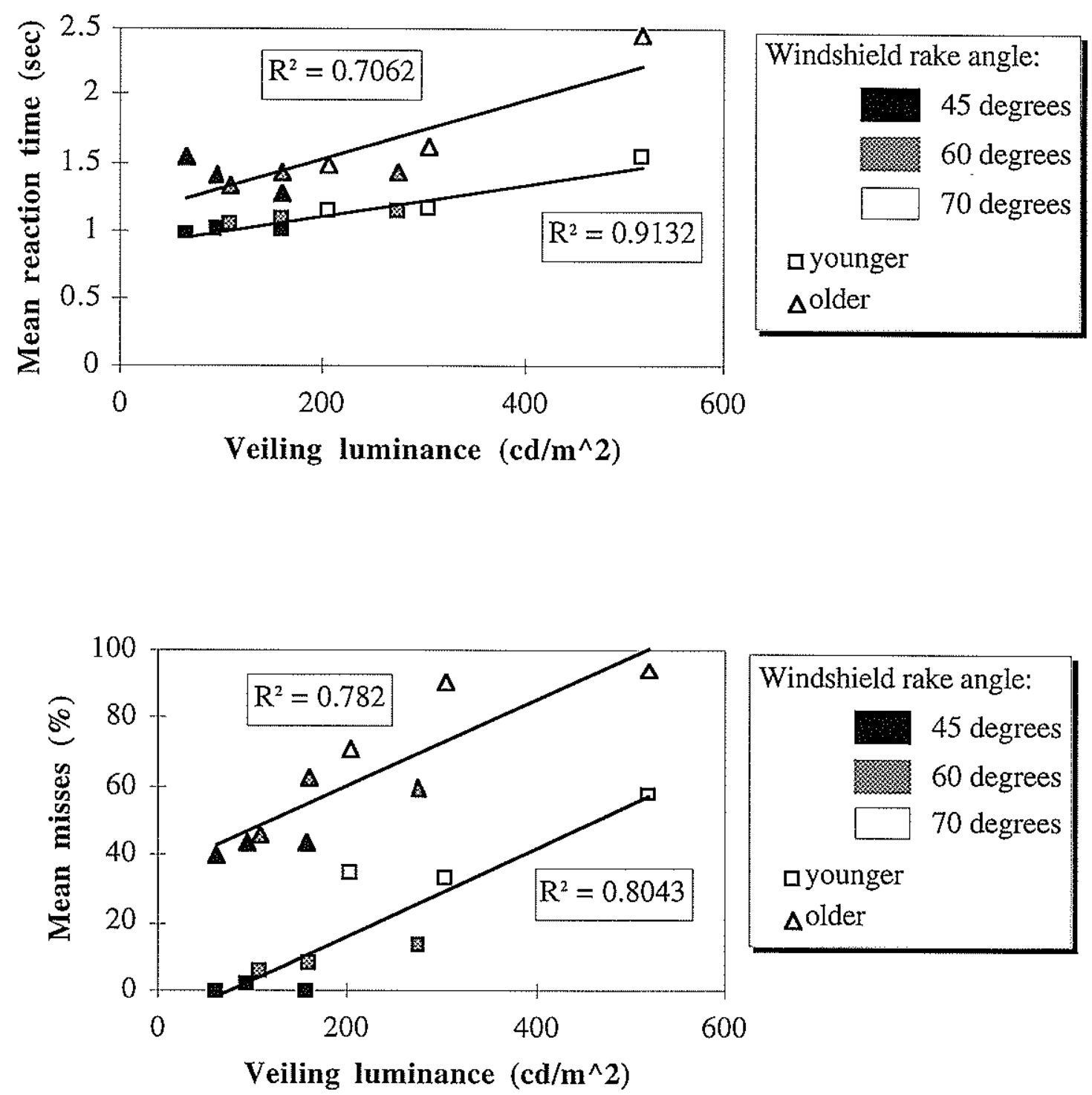

Figure 19. Mean reaction time to the high-contrast pedestrian (top panel) and mean misses of the low-contrast pedestrian (bottom panel) as a function of the calculated veiling luminance. The nine values of veiling luminance on the horizontal axis correspond to all combinations of the three rake angles and three dashboard reflectances. The three points within each group corresponding to a single rake angle differ in dashboard reflectance, with higher reflectance always corresponding to higher veiling luminance. The lines are the best fitting linear regressions. 


\section{DISCUSSION}

The results of this study indicate that both windshield rake angle and dashboard reflectance have measurable effects on visual performance. The reduced contrast produced by these conditions can decrease visual performance, especially for older drivers.

Veiling glare increases with larger windshield rake angles, especially with rake angles larger than 60 degrees. Subjects needed more time to detect high-contras objects (see Figure 8), and they had more misses in detecting low-contrast objects (see Figure 9). This empirical relationship is consistent with the fact that reflectance increases markedly with incidence angles larger than 60 degrees (see Figure 3).

The effect of veiling glare on the ability to see is particularly pronounced if a large rake angle is combined with high dashboard reflectance (see Figure 10). This relationship should be recognized when selecting dashboard material for passenger cars or vans with large windshield rake angles. Consequently, the results of this study suggest that negative effects of the large rake-angle condition could be compensated for with a low dashboard reflectance (see Figure 10).

The perceptual consequences of decreased contrast sensitivity of older persons became evident when older subjects had to detect low-contrast objects (see Figure 12), or when extreme veiling glare reduced the contrast of otherwise high-contrast objects (see Figure 11). The implication is that older drivers are more at risk in situations involving high levels of veiling glare. Interestingly, older subjects overestimated their own ability to see under large rake-angle conditions (see Figure 15).

Overall, however, the ratings show that subjects are reasonably well aware of the veiling glare problem, because the rating data corresponded well with the objective reaction time data (e.g., compare Figure 8 or 9 with Figure 15). Both subject groups gave their lowest visibility ratings for the condition involving the largest rake angle and the highest dashboard reflectance.

The results of this study concerning the influence of dashboard reflectance compare well with those from a laboratory study by Sauter et al. (1992). On the other hand, contrary to the findings of Sauter et al. (1992), the present study found a strong effect of rake angle.

This study concentrated on the influence of windshield rake angle and dashboard reflectance on driver visual performance. Both conditions can contribute to daytime veiling glare. The possible influence of other factors such as dashboard gloss, texture, and inclination angle remain to be empirically ascertained. 


\section{REFERENCES}

Edson, L. (1992). Veiling Glare: What Factors Create It and How to Use these Factors to Predict the Performance of a Vehicle Design. (SAE Technical Paper Series No. 940646). Warrendale, PA: Society of Automotive Engineers.

Fed. Std. No. 515/3a (1967). Instrument panel instrument and control devices for automotive vehicles. Washington, D.C.: GSA.

Käppler, W.D., Godthelp, J., v.Randwijk, M.J., and Ruijs, P.A. (1992). Methodology for predicting car and truck handling assessments. In Proc. XXIV FISITA Congress Total Vehicle Dynamics Volume 1 (pp. 25-34). London: Mechanical Engineering Publications (FISITA No. 925044).

Kaufman, J.E. and Christenson, J.F. (1984). IES Lighting Handbook 1984 Reference Volume. New York: Illuminating Engineering Society of North America.

Kirk, R.E. (1982). Experimental design: Procedures for the behavioral sciences (2nd ed.). Pacific Grove: Brooks/Cole Publishing Company.

McCluney, W.R. (1994). Introduction to Radiometry and Photometry. Boston: Artech House.

NHTSA (1991). Report to Congress on Tinting of Motor Vehicle Windows. Washington, D.C.: NHTSA.

Pitrella, F.D. and Käppler, W.D. (1988). Identification and Evaluation of Scale Design Principles in the Development of the Extended Range, Sequential Judgment Scale. (Report No. 80). Wachtberg: Research Institute for Human Engineering.

Sauter, J.L., Bow, N., LaDriere, M.L., and Parman, S.C. (1992). The effects of Instrument Panel Top Color and Windshield Angle on Veiling Glare. (SAE Technical Paper Series No. 940646). Warrendale, PA: Society of Automotive Engineers.

Sivak, M., Campbell, K.L., Schneider, L.W., Sprague, J.K., Streff, F.M., and Waller, P.F. (1995). The safety and mobility of older drivers: What we know and promising research issues. UMTRI Research Review, 26, 1-21. 
Storms, C.D. (1994). Instrument Panel Coatings to Reduce Veiling Glare and for Decorative Effects (SAE Technical Paper Series No. 940646). Warrendale, PA: Society of Automotive Engineers.

Wyszecki, G. and Stiles, W.S. (1982). Color Science: Concepts and Methods, Quantitative Data and Formulae (2nd ed.). New York: John Wiley \& Sons.

49 Code of Federal Regulations Part 571.107 (1994). Federal Motor Vehicle Safety Standard No. 107; Reflecting Surfaces. Washington, D.C.: GPO. 